\title{
Portal vein reconstruction with interposition of cryopreserved aortic graft: A case report and literature review
}

\author{
NICOLAE BACALBASA $^{1,2}$, IRINA BALESCU ${ }^{3}$, CAMELIA DIACONU $^{4,5}$, BOGDAN SOCEA ${ }^{6,7}$, \\ FLORENTINA GHERGHICEANU $^{8}$, OVIDIU STIRU ${ }^{9,10}$ and VLADISLAV BRASOVEANU ${ }^{1}$ \\ ${ }^{1}$ Department of Visceral Surgery, Center of Excellence in Translational Medicine, \\ 'Fundeni' Clinical Institute, 022328 Bucharest; ${ }^{2}$ Department of Obstetrics and Gynecology, \\ 'Carol Davila' University of Medicine and Pharmacy, 020021 Bucharest; ${ }^{3}$ Department of Surgery, \\ 'Ponderas' Academic Hospital, 021188 Bucharest; ${ }^{4}$ Department of Internal Medicine, 'Carol Davila' \\ University of Medicine and Pharmacy, 020021 Bucharest; ${ }^{5}$ Department of Internal Medicine, \\ Clinical Emergency Hospital of Bucharest, 014461 Bucharest; ${ }^{6}$ Department of Surgery, \\ 'Carol Davila' University of Medicine and Pharmacy, 020021 Bucharest; ${ }^{7}$ Department of Surgery, \\ 'Sf. Pantelimon' Emergency Hospital, 021659 Bucharest; ${ }^{8}$ Department of Marketing and \\ Medical Technology, 'Carol Davila' University of Medicine and Pharmacy, 020021 Bucharest; \\ ${ }^{9}$ Department of Cardiovascular Surgery, 'Prof. Dr. C.C. Iliescu' Emergency Institute for \\ Cardiovascular Diseases, 022322 Bucharest; ${ }^{10}$ Department of Cardiovascular Surgery, \\ 'Carol Davila' University of Medicine and Pharmacy, 020021 Bucharest, Romania
}

Received September 3, 2021; Accepted October 5, 2021

DOI: $10.3892 / \mathrm{etm} .2021 .11107$

\begin{abstract}
Pancreatic cancer is one of the most aggressive malignancies with poor rates of survival especially in the event radical procedures are not feasible. However, improvements in surgical techniques have led to the successful association of vascular resection followed by reconstruction without a significant increase in the rates of postoperative complications. In the present article, we present the case of a 49-year-old patient diagnosed with pancreatic head cancer invading the portal vein. After discussing with the patient the risks and the benefits of the surgical procedure, the patient was submitted to pancreatoduodenectomy en bloc with portal vein resection while the continuity of the portal vein was reestablished by using a cadaveric graft originating from the abdominal aorta. The postoperative outcome was uneventful. In conclusion, in selected cases, arterial cadaveric grafts may be used in order to establish the continuity of the portal vein with good results. However, it should be emphasized that these are demanding procedures which should be carefully analyzed before deciding upon the opportunity for performing them.
\end{abstract}

Correspondence to: Dr Nicolae Bacalbasa, Department of Obstetrics and Gynecology, 'Carol Davila' University of Medicine and Pharmacy, 8 Eroilor Sanitari Street, 020021 Bucharest, Romania E-mail: nicolae_bacalbasa@yahoo.ro

Key words: pancreatic cancer, portal vein resection, cadaveric graft, abdominal aorta, venous reconstruction

\section{Introduction}

Improvements in the field of surgical oncology along with advancements reported to date in medical oncology and radiotherapy have led to an increase in the rates of resectability of pancreatic head tumors and therefore, to an increase in the proportion of patients reporting a significant benefit in terms of survival $(1,2)$. However, the close anatomic relationship between the pancreatic head and the surrounding vascular structures is still responsible for the presence of local invasion in a significant number of cases (3). In this respect, attention was focused on determining whether the presence of such a vascular encasement should be considered as a formal contraindication for resection or if, in certain cases, vascular resection makes sense in order to improve the long-term outcomes (4). Promising results have been reported to date in regards to portal vein resection followed by reconstruction; in such cases, similar rates of long-term survival have been reported when compared to standard pancreatic resections (5). Therefore, portal vein resection is no longer a formal contraindication for resection and attention was focused on identifying the best graft for portal reconstruction.

\section{Case report}

After obtaining the approval of the Ethics Committee of 'Fundeni' Clinical Institute (no. 752/2020), data of the patient were retrospectively reviewed.

The 49-year-old patient with no significant medical history was investigated for diffuse abdominal pain, weight loss of $7 \mathrm{~kg}$ in the last three months and jaundice. The biochemical 
tests revealed the presence of cholestasis, with serum levels of total bilirubin of $9.2 \mathrm{mg} / \mathrm{dl}$ and direct bilirubin of $7.5 \mathrm{mg} / \mathrm{dl}$, cytolysis, with aspartate aminotransferase (AST) of $344 \mathrm{U} / 1$ and alanine aminotransferase of $599 \mathrm{U} / 1$. Meanwhile the serum levels of cancer antigen (CA19-9) were significantly increased (CA19-9, $425 \mathrm{U} / \mathrm{ml})$. The patient was submitted to magnetic resonance imaging (MRI) which demonstrated the presence of a $4 / 5 / 3 \mathrm{~cm}$ pancreatic head mass with no demarcation line with the portal vein on a distance of $2.2 \mathrm{~cm}$. No sign of invasion of the hepatic pedicle or of the superior mesenteric vessels were observed. The patient was further submitted to an endoscopic ultrasound in order to retrieve a biopsy which demonstrated the presence of a moderately differentiated pancreatic adenocarcinoma. Due to the presence of a good general status and due to the absence of other signs of unresectability, the patient was submitted to per primam resection, a pancreatoduodenectomy en bloc with portal vein resection being performed. The length of the resected portal vein was of $3.5 \mathrm{~cm}$ so an end to end anastomosis was not feasible. Due to this reason the decision for using a cadaveric graft was taken, the continuity of the portal vein being re-established by placing a cadaveric cryopreserved abdominal aorta graft measuring $3.5 \mathrm{~cm}$. The anastomosis between the portal vein and the cadaveric aortic graft were performed by using a running suture of polypropylene 5-0 (Fig. 1). The duration of the surgery was 230 min while the estimated blood loss was of $200 \mathrm{ml}$; no intraoperative complications were encountered. During the perioperative period, the patient was submitted to low-molecular heparin injection, the patency of the graft being demonstrated by Doppler ultrasound which was performed on the second and seventh postoperative days. The patient was discharged on the eight postoperative day and received recommendation to continue the administration of low-molecular heparin for the next 30 days. The histopathological studies demonstrated the presence of a moderately differentiated pancreatic adenocarcinoma invading the portal vein on a total length of $2.8 \mathrm{~cm}$; meanwhile, all the resection margins of the specimen were free of disease. At the one month follow-up, the patient reported a good general condition and was deferred to the oncology department in order to be submitted to adjuvant chemotherapy.

\section{Discussion}

Initially performed in the late 1950 's, pancreatic head resection en bloc with venous resection and reconstruction were considered at that moment as unjustified due to the high rates of perioperative complications. Therefore, venous invasion has been considered for a long period of time as a formal contraindication for surgery; however recent meta-analyses came to demonstrate that the method can be safely applied without a significant increase in perioperative morbidity and meanwhile, with significant benefits in terms of survival (5-8). These similar rates of long-term survival which have been reported after standard pancreatoduodenectomy when compared to pancreatoduodenectomy en bloc with venous resection have been explained by the fact that local venous invasion is rather the sign of a locally advanced disease and not of a biologically aggressive lesion (9). Meanwhile, improvements in the surgical techniques concerning vascular reconstruction, of the

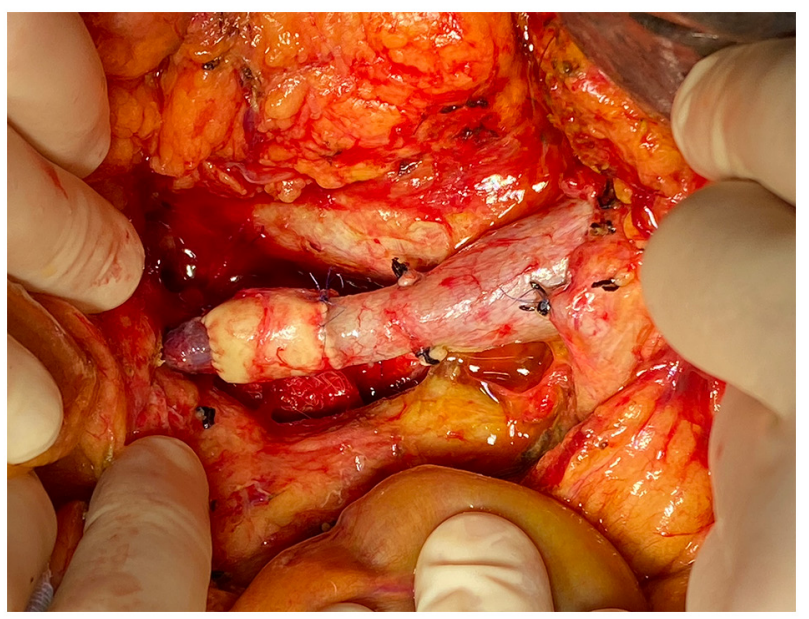

Figure 1. Final aspect of the portal vein reconstruction by using a cadaveric graft of the abdominal aorta.

perioperative and postoperative management have led to the successful incorporation of such resections as part of pancreatic surgery (10-16).

According to the extent of local invasion of the portal vein, different types of procedures have been proposed to date; therefore, resection of the portal vein might be a lateral one or a circumferential one (17-19). Cases in which the estimated degree of narrowing after lateral resection is larger than $30 \%$ of the portal lumen are rather submitted to a circumferential resection than to a lateral one followed by lateral venorrhaphy (18); meanwhile, in cases in which a circumferential resection is needed, the type of reconstruction is to be established depending on the length of the resected segment as well as of the diameter of the two venous stumps $(16,17)$. In cases in which the extent of portal vein resection is limited and the two stumps have similar diameters, an end to end anastomosis might be the option of choice; generally it is estimated that defects shorter than $2 \mathrm{~cm}$ are suitable for an end to end anastomosis (18). Meanwhile cases in which the resulting defect does not allow an end to end anastomosis, a graft may be needed in order to re-establish the venous continuity. In order to minimize the risks of perioperative complications, multiple types of reconstructions have been proposed including: autologous vein, cadaveric arterial or venous grafts, bovine pericardial or synthetic grafts such as Gore-Tex, polytetrafluoroethylene or Dacron prostheses (18). Whenever a venous graft of a synthetic prosthesis is used, there is a significant risk of graft thrombosis which might reach $17 \%$ of cases, depending on the extent of resection, timing and graft harvesting and type of reconstruction (19-22).

When it comes to the utility of arterial cadaveric grafts for vascular reconstructions, the method has been initially implemented in vascular surgery in order to provide different types of arterial reconstructions. Cryopreserved aortic graft was successfully implemented for aortic reconstruction and have proven to have significant benefits; therefore, according to Harlander-Locke et al, this method is associated with decreased risks of graft infection, aneurysm formation and limb loss. The authors conducted a multicenter retrospective study which involved 220 patients and demonstrated that at a 5-year follow up $97 \%$ of cases reported a patent aortic 
graft (19). Once the method proved its efficacy in vascular surgery, it was also successfully implemented in visceral surgery. Therefore, it avoids the risks associated with the use of synthetic allografts such as Dacron or Gore-Tex by diminishing the infectious risks. In a study conducted by Mascoli et al, aortic graft reconstruction of the portal vein was successfully reported in three cases; two patients benefited from a thoracic aorta graft while the third one benefited from an abdominal aorta graft, the median length of the graft being $6 \mathrm{~cm}$. Meanwhile the authors reported the superiority of the method when compared to venous graft reconstruction due to the fact that arterial grafts have a lower risk of developing postoperative complications such as thrombosis, stenosis or infection (22). Therefore, it seems that arterial grafts are more resistant to surgical manipulation, do not have valves, are more effective in reducing the occlusion risk due to external visceral compression and are associated with a lower risk of graft infection $(23,24)$.

In conclusion, cryopreserved cadaveric arterial grafts appear to be safely used in portal vein reconstruction after pancreatoduodenectomy en bloc with portal vein resection for locally advanced pancreatic cancer. Therefore, arterial grafts seem to have certain advantages when compared to venous grafts or synthetic prosthesis such as a lower risk of postoperative graft infection, thrombosis or stenosis. However, findings concerning this method have been scarcely reported so far, or only for a small number of cases. Thus, larger studies are necessary in order to standardize it and to analyze which cases could benefit most after this type of reconstruction.

\section{Acknowledgements}

Not applicable.

\section{Funding}

This research received no external funding.

\section{Availability of data and materials}

Data are available at request from the corresponding author.

\section{Authors' contributions}

NB, IB and OS contributed to the conception and design of the present study. $\mathrm{CD}, \mathrm{BS}, \mathrm{FG}, \mathrm{IB}, \mathrm{VB}$ consulted the relevant references and performed the literature data collection. IB and NB wrote the first draft of the manuscript. VB revised the manuscript. All authors read and approved the final manuscript for publication.

\section{Ethics approval and consent to participate}

The Ethics Committee of 'Fundeni' Clinical Institute approved the study (no. 752/2020).

\section{Patient consent for publication}

Patient consent for publication was obtained and signed by the patient on 21/04/2021.

\section{Competing interests}

The authors declare that they have no competing interests.

\section{References}

1. Smeenk HG, van Eijck CH, Hop WC, Erdmann J, Tran KC, Debois M, van Cutsem E, van Dekken H, Klinkenbijl JH and Jeekel J: Long-term survival and metastatic pattern of pancreatic and periampullary cancer after adjuvant chemoradiation or observation: Long-term results of EORTC trial 40891. Ann Surg 246: 734-740, 2007.

2. Varadhachary GR, Tamm EP, Abbruzzese JL, Xiong HQ Crane $\mathrm{CH}$, Wang H, Lee JE, Pisters PWT, Evans DB and Wolff RA: Borderline resectable pancreatic cancer: Definitions, management, and role of preoperative therapy. Ann Surg Oncol 13: 1035-1046, 2006.

3. Peparini N and Chirletti P: Mesopancreas: A boundless structure, namely R1 risk in pancreaticoduodenectomy for pancreatic head carcinoma. Eur J Surg Oncol 39: 1303-1308, 2013.

4. Beger HG, Rau B, Gansauge F, Poch B and Link KH: Treatment of pancreatic cancer: Challenge of the facts. World J Surg 27: 1075-1084, 2003.

5. Weitz J, Kienle P, Schmidt J, Friess H and Büchler MW: Portal vein resection for advanced pancreatic head cancer. J Am Coll Surg 204: 712-716, 2007.

6. Tseng JF, Raut CP, Lee JE, Pisters PW, Vauthey JN, Abdalla EK, Gomez HF, Sun CC, Crane CH, Wolff RA and Evans DB: Pancreaticoduodenectomy with vascular resection: Margin status and survival duration. J Gastrointest Surg 8: 935-950, 2004.

7. Siriwardana HP and Siriwardena AK: Systematic review of outcome of synchronous portal-superior mesenteric vein resection during pancreatectomy for cancer. Br J Surg 93: 662-673, 2006.

8. Hartel M, Niedergethmann M, Farag-Soliman M, Sturm JW, Richter A, Trede M and Post S: Benefit of venous resection for ductal adenocarcinoma of the pancreatic head. Eur J Surg 168: 707-712, 2002.

9. Leach SD, Lee JE, Charnsangavej C, Cleary KR, Lowy AM, Fenoglio CJ, Pisters PW and Evans DB: Survival following pancreaticoduodenectomy with resection of the superior mesenteric-portal vein confluence for adenocarcinoma of the pancreatic head. Br J Surg 85: 611-617, 1998.

10. Bacalbasa N, Brezean I, Anghel C, Barbu I, Pautov M, Balescu I and Brasoveanu V: Successful resection and vascular ligation of a large hepatic artery aneurysm-a case report and literature review. In Vivo 31: 979-982, 2017.

11. Bacalbasa N, Brezean I, Anghel C, Barbu I, Pautov M, Balescu I and Brasoveanu V: Management of a fulminant upper gastrointestinal bleeding exteriorized through hemobilia due to arteriobiliary fistula between the common bile duct and a right hepatic artery aneurysm-a case report. In Vivo 31: 983-989, 2017.

12. Braşoveanu V, Dumitraşcu T, Bacalbaşa N and Zamfir R: Splenic artery used for replaced common hepatic artery reconstruction during pancreatoduodenectomy-a case report. Chirurgia (Bucur) 104: 499-504, 2009.

13. Brasoveanu V, Anghel C, Barbu I, Pautov M, Ionescu MI, Motthor M, Balescu I, Dima S and Bacalbasa N: Pancreatoduodenectomy en bloc with portal and superior mesenteric artery resection-a case report and literature review. Anticancer Res 35: 1613-1618, 2015.

14. Bacalbasa N, Balescu I, Tanase A, Pautov M, Brezean I, Vilcu M and Brasoveanu V: Spleno-pancreatectomy en bloc with parcelar gastrectomy for splenic artery aneurysm-a case report and literature review. In Vivo 32: 915-919, 2018

15. Bacalbasa N, Balescu I, Tanase A, Brezean I, Vilcu M and Brasoveanu V: Successful resection of a non-functional paraganglioma with celiac trunk invasion followed by common hepatic artery reimplantation-a case report and literature review. In Vivo 32: 911-914, 2018.

16. Ishikawa O, Ohigashi H, Imaoka S, Furukawa H, Sasaki Y, Fujita M, Kuroda C and Iwanaga T: Preoperative indications for extended pancreatectomy for locally advanced pancreas cancer involving the portal vein. Ann Surg 215: 231-236, 1992. 
17. Kleive D, Berstad AE, Verbeke CS, Haugvik SP, Gladhaug IP, Line PD and Labori KJ: Cold-stored cadaveric venous allograft for superior mesenteric/portal vein reconstruction during pancreatic surgery. HPB (Oxford) 18: 615-622, 2016.

18. Glebova NO, Hicks CW, Piazza KM, Abularrage CJ Cameron AM, Schulick RD, Wolfgang CL and Black JH III: Technical risk factors for portal vein reconstruction thrombosis in pancreatic resection. J Vasc Surg 62: 424-433, 2015.

19. Harlander-Locke MP, Harmon LK, Lawrence PF, Oderich GS McCready RA, Morasch MD, Feezor RJ; Vascular Low-Frequency Disease Consortium, Zhou W, Bismuth J, et al: The use of cryopreserved aortoiliac allograft for aortic reconstruction in the United States. J Vasc Surg 59: 669-674, 2014.

20. Smoot RL, Christein JD and Farnell MB: An innovative option for venous reconstruction after pancreaticoduodenectomy: The left renal vein. J Gastrointest Surg 11: 425-431, 2007.
21. Fleming JB, Barnett CC and Clagett GP: Superficial femoral vein as a conduit for portal vein reconstruction during pancreaticoduodenectomy. Arch Surg 140: 698-701, 2005.

22. Mascoli C, D'Ambra M, Casadei R, Ricci C, Taffurelli G, Ancetti S, Stella A, Minni F and Freyrie A: Portal/superior mesenteric vein reconstruction during pancreatic resection using a cryopreserved arterial homograft. Dig Surg 32: 284-290, 2015.

23. Ravikumar R, Sabin C, Abu Hilal M, Bramhall S, White S, Wigmore S, Imber CJ and Fusai G; UK Vascular Resection in Pancreatic Cancer Study Group: Portal vein resection in borderline resectable pancreatic cancer: A United Kingdom multicenter study. J Am Coll Surg 218: 401-411, 2014.

24. Dodd PD: What tissue bankers should know about the use of allograft blood vessels. Cell Tissue Bank 11: 3-11, 2010. 